\title{
Transition of MHD Kink-Stability Properties between Line-Tied and Non-Line-Tied Boundary Conditions
}

\author{
X. Sun, ${ }^{1}$ T. P. Intrator, ${ }^{1}$ L. Dorf, ${ }^{1}$ I. Furno, ${ }^{1}$ and G. Lapenta ${ }^{1,2}$ \\ ${ }^{1}$ Los Alamos National Laboratory, Mail Stop E526, Los Alamos, New Mexico 87545, USA \\ ${ }^{2}$ Centrum voor Plasma-Astrofysica, Katholieke Universiteit Leuven, Belgium \\ (Received 9 November 2007; revised manuscript received 16 January 2008; published 23 May 2008)

\begin{abstract}
Magnetic flux tubes or flux ropes in plasmas are important in nature and the laboratory. Axial boundary conditions strongly affect flux rope behavior, but this has never been systematically investigated. We experimentally demonstrate for the first time axial boundary conditions that are continuously varied between ideal magnetohydrodynamic (MHD) line-tied (fixed) and non-line-tied (free). In contrast with the usual interpretation that mechanical plasma motion is MHD line-tied to a conducting boundary, we constrain boundary plasma motion to cause the line-tied condition.
\end{abstract}

Magnetic fields that confine plasmas and electrical currents, both in nature and in the laboratory, are tubular structures, known as flux tubes or flux ropes. The magnetohydrodynamic (MHD) model is frequently used to explain plasma behavior of ensembles of magnetic field lines, including their relaxation, stability, and wave characteristics. The flux rope is an important concept for realistic plasma objects with structure and geometry. Flux tubes can be periodic, for instance in toroidal devices such as a tokamak. Or they can have finite length in the axial direction, such as flux tubes in the solar corona [1,2], during spheromak startup [3], $Z$ pinches [4], plasma thrusters [5], and possibly astrophysical jets $[6,7]$. Those flux tubes with finite length must have an axial boundary.

In this letter, we experimentally demonstrate for the first time axial boundary conditions (BC) that can be continuously adjusted between line-tied (LT) and non-line-tied (NLT) conditions. We show that the BC strongly affects the external kink mode, and use this fact to discriminate between different BC's.

Even though the LT condition has been invoked nearly 40 years ago for coronal flux ropes [8], there has been surrounding controversy [9]. In the commonly accepted ideal MHD view, the LT axial boundary condition means that each fluid element is "frozen" to the local magnetic field. Therefore a LT ideally conducting plasma should follow LT magnetic field lines which are immobile at the end of the flux tube. However for a highly resistive boundary, the plasma end can be free to move. This can be described as a perfectly non-line-tied (PNLT) boundary. It is useful to pose the $\mathrm{BC}$ mathematically as a linear combination of flux tube displacement and tilt angle.

In the following $\zeta\left(=\zeta_{x}+i \zeta_{y}\right)$ represents a complex scalar transverse displacement of an MHD fluid element from its equilibrium position, for example, in Fig. 1(a). We define the plasma column pitch as $\partial \zeta / \partial z$. For the frozen flux condition, magnetic field lines on a toroidal flux surface can be ergodic and effectively trace out an irrational flux surface, or fall on a rational surface. Nontoroidal field lines can be finite and helically periodic. For external MHD modes the field line pitch is characterized by $k_{o}=$ $B_{\theta} / a B_{z}$, where $a$ is the radius of the flux rope and $B_{\theta}(a)$, $B_{z}(a)$ are azimuthal and axial magnetic field strengths evaluated at radius $r=a[6]$. Then the $\mathrm{BC}$ of the displaced
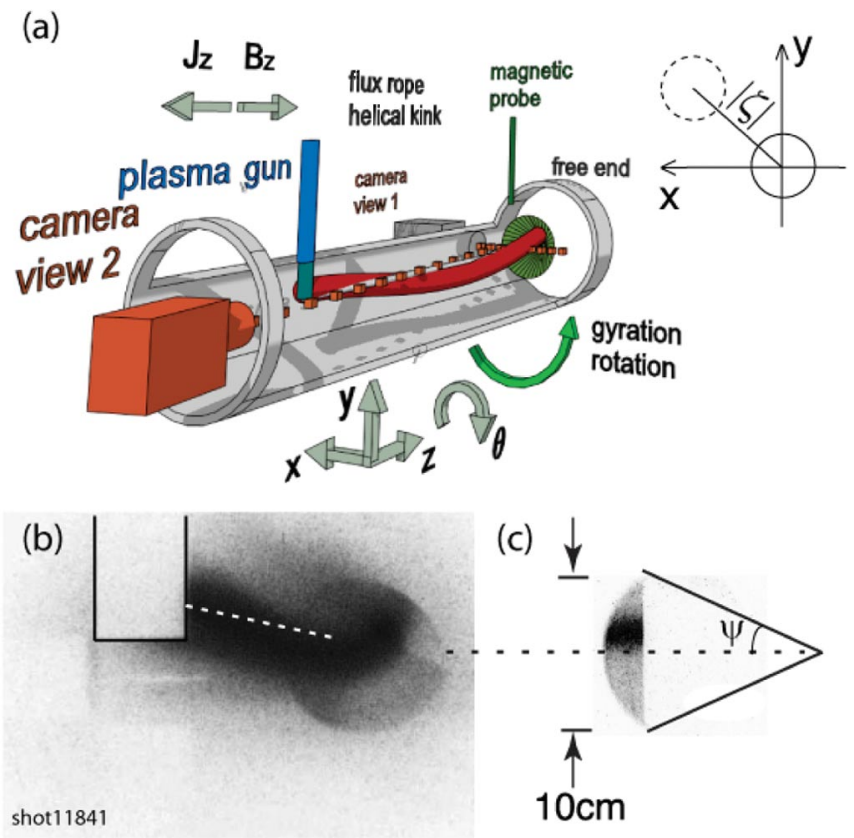

FIG. 1 (color). (a) Perspective drawing of RSX experimental setup and coordinates with 0.8 turns of writhe, displacement $|\zeta|=2 \mathrm{~cm}\left(z=l_{z}\right)$. The definition of $\zeta$ is shown in the right-top corner. It is the complex scalar displacement of the perturbed plasma column (circle with dashed line) from its initial position (circle with solid line). (b) $H_{\alpha}$ emission camera image, $300 \mathrm{nsec}$ gate, from view 2 , showing the flux rope striking a cone $(\psi=$ $\left.22.5^{\circ}\right), I_{p}=200 \mathrm{Amp}>I_{\mathrm{th}}, B_{z}=100 \mathrm{G}$, substantially above the threshold current. Absent the kink, the flux rope would follow the dashed lines (c) Side-on flux rope image and cone schematic, silhouetted by the round vacuum window from view 1 at the time of maximum $\xi$ excursion. 
plasma column can be expressed as $\partial \zeta / \partial z(z=0)=$ $\partial \zeta / \partial z(z=l)$, where $l$ is the column length. The $\partial \zeta / \partial z$ can increase the field line pitch or equivalently make $B_{\theta}$ larger, and thus it can enhance the perturbation [10].

On the other hand, in a nonperiodic system flux tubes with finite length can have line-tied (LT) ends [11,12], where the displacements $\zeta(z=0)=\zeta(z=l)$ are constrained to remain fixed. If there is a fixed end $\zeta(z=0)$ plus a PNLT end, then the BC becomes a weighted combination of the aforementioned BC's: $\partial \zeta / \partial z+i\left(k_{0} / 2\right) \zeta=$ $0(z=l)[13,14]$.

The plasma motion at the boundary and therefore the BC can affect the global plasma status far from the boundary. In toroidal systems there can be one unidirectional (e.g., Alfvén) wave, but in a finite cylindrical system, there can be reflections. The boundary condition can radically affect the nature of the Alfven spectrum [1], as well as the stability properties $[14,15]$. For instance field line curvature and thus the $(\vec{B} \cdot \vec{\nabla}) \vec{B}$ MHD restoring force is reduced when the end becomes non-line-tied and $\zeta$ is nonzero. A systematic investigation of the role of BC's would be useful to study the effects on MHD stability, relaxation, and waves as flux tubes gradually become constrained by more and more line-tying at the ends.

We investigate this by creating a linear flux rope that is terminated at a shaped axial boundary. With this change of shape the axial BC's are continuously varied between LT and PNLT conditions. We experimentally demonstrate this transition with magnetic probe measurements of the external kink instability threshold. The Kruskal-Shafranov (KS) threshold [10] for kink instability corresponds to the linetied $\mathrm{BC}$, and also to the toroidally periodic case. A change of geometry and hence the $\mathrm{BC}$ can reduce the kink threshold to approximately half the KS prediction, which corresponds to the PNLT case.

Experiments were conducted in the reconnection scaling eXperiment (RSX) [16] which uses a plasma gun to generate a plasma current channel embedded in a background magnetic guide field $B_{z}$, with electron temperature $T_{e} \approx$ $5-14 \mathrm{eV}$, plasma density $n \approx 1-3 \times 10^{13} \mathrm{~cm}^{-3}$, column radius $a \sim 2.0 \mathrm{~cm} \mathrm{[6]} \mathrm{and} \mathrm{length} l_{z} \approx 96 \mathrm{~cm}$. The plasma current $I_{p}$ can be adjusted with an external anode extraction voltage, $V_{b}$. When $I_{p} / B_{z}$ is large enough, the kink is driven unstable, and this threshold can be compared for instance with the KS value of $\mu_{0} I_{\mathrm{KS}} / \Phi>4 \pi / l_{z}$, where $\Phi=B_{z} \pi a^{2}$ is the axial flux. The gun end $\mathrm{BC}$ has fixed $\zeta(z=0)=0$, and the external anode end $\mathrm{BC}$ has an insertable cone with the wide end facing the gun, and half angle $0<\psi<\pi / 2$. Figure 1(a) is a perspective schematic of the RSX experimental setup and coordinates. Figure 1(b) shows an end-on, perspective, $H_{\alpha}$ image of the whole kinked plasma column which is a helical distortion wrapped around a conical surface $[6,14,17]$, between gun and cone anode positions. The side view image in Fig. 1(c) from view 1 [see Fig. 1(a)] shows the flux rope end and a superposed schematic cone outline. $B_{z}$ is
100 Gauss for all these experiments. Clearly the plasma is displaced from the center dashed line; i.e., the displacement of the plasma footpoint at the boundary is nonzero.

The nonzero displacement during a finite time indicates a nonzero force tangent to the boundary. For the experiment shown in this Letter, there is a conducting boundary partially insulated by a sheath. As shown in previous work [14], the plasma-sheath boundary provides an electric field $\vec{E}$ and a tangential force that drives a plasma footpoint movement $\frac{\partial \vec{\xi}}{\partial t}=\frac{\vec{E} \times \vec{B}}{B^{2}}$, where $\vec{\xi}$ is the vector displacement. Thus, in the following we first present the experimental evidence that there exists a sheath and then we argue there is a tangential electric field which is also affected by the boundary shape, so that this constraint leads to the BC. Figure 2(a) shows the electric circuit including capacitors, switching, guns, and high impedance current source for the flux rope. Figure 2(b) shows experimentally measured data points. The plasma potential data indicate an overall plasma electrostatic electric field $\left(E_{z} \approx 2-3 \mathrm{~V} / \mathrm{m}\right)$ that points from the anode to gun, and the external anode bias potential $\left(V_{b}\right)$ is consistent with an electron repelling boundary sheath. $V_{b}$ is then determined both by bias bank voltage, the plasma-sheath characteristic, and hence the plasma current. This makes RSX a unique selfconsistent system with a current source boundary, driven by plasma currents and particles, rather than an imposed electric field. The endplate current-voltage trace in Fig. 2(c) [18] corresponds to a sheath that repels plasma electrons for $I_{p}<200$ Amp.

If the magnetic field is normal to the boundary $(\psi=$ $90^{\circ}$ ) [13,19] one can write the incident current density from plasma to sheath as

$$
j_{z}(\phi)=e n\left(u-v_{\mathrm{Te}} e^{-e \phi / T_{e}}\right),
$$

where $\phi=V_{b}-V_{p}, V_{p}$ is the plasma potential, $u$ and $v_{\mathrm{Te}}$ are the ion flow speed (on the order of ion sound speed) and electron thermal speed [13], $e>0$ is the absolute value of the electron charge, and $T_{e}$ is the electron temperature. The first order perturbed current density equation can be easily found to be: $\delta j_{z}=\left(e n u-j_{0}\right) \frac{e \delta \phi}{T_{e}}$, and $j_{0}$ is the zeroth order quantity from Eq. (1) [13]. Thus a perturbation in current density can yield a perturbed sheath potential $\delta \phi$. If this perturbation is $\theta$ dependent, as would be case for the kink mode, then $\delta \phi$ is different around the plasma circumference. In other words, a tangential sheath electric field appears that can drive the plasma motion at the boundary. For a flat endplate $\left(\psi=90^{\circ}\right)$ without any constraints on the plasma movement, the plasma in the sheath region can move independently of the magnetic field lines (PNLT) if the sheath resistive decay time (column inductance or sheath resistance) is shorter than the Alfven time [13].

As it has been noted for tokamak divertors [20-22], when a magnetic field intercepts an obliquely tilted boundary, the sheath structure reflects nearly all the electrons, passes ions and has multiple scale lengths besides the Debye length, including the ion and electron gyroradii. A 
(a)

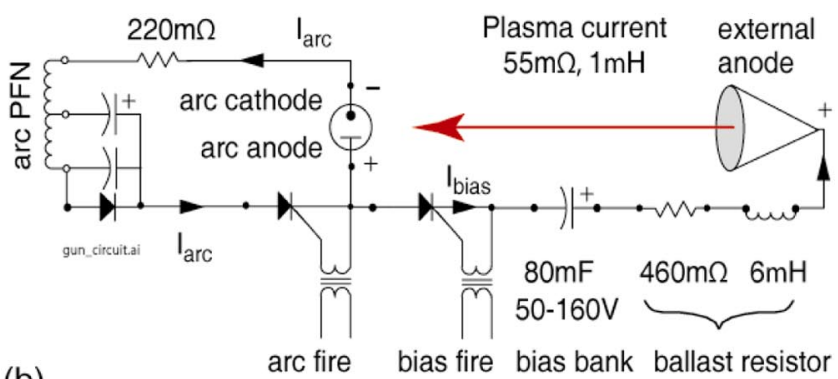

(b)

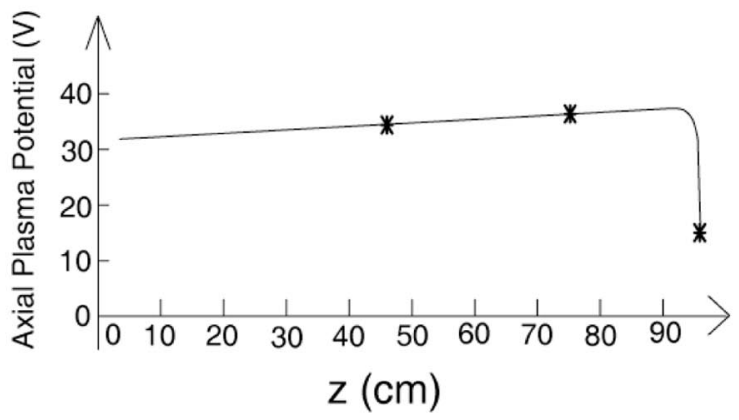

(c)

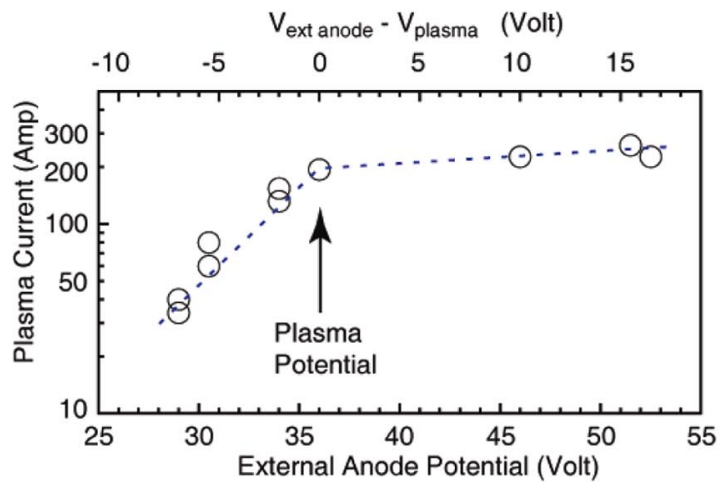

FIG. 2 (color online). (a) Plasma gun circuit schematic, showing arc pulse forming network, plasma current source impedance, but omitting charge and dump circuits. (b) Measured potential values are indicated with an asterisk in the plasma $(z=$ $46,74 \mathrm{~cm})$ and at the external anode $(z=92 \mathrm{~cm})$ and the likely axial plasma potential profile is indicated with the solid line. (c) Current-Voltage characteristic of the conical anode during the flat top of the current vs time history. Estimated plasma potential is consistent with an electron repelling external anode sheath for the $I_{p}<200$ Ampere data in Figs. 3 and 4.

magnetic subsheath forms with ion gyro radius length scale, which supports a potential drop that depends on $\psi$ (see Eq. 2.109 in [19] and [20]), which can modify Eq. (1), and the consequent electric field. A tilted boundary therefore should affect the tangential electric field and BC.

We have used different conical endplates to systematically investigate the constraints on the boundary conditions ranging from a narrow cone $\left(\psi=15^{\circ}\right)$ to a flat plate $(\psi=$ $90^{\circ}$ ). For $\psi=90^{\circ}, 75^{\circ}, 60^{\circ}, 45^{\circ}, 22.5^{\circ}$, and, $15^{\circ}$ the increasingly constrained flux rope displacement $\mathrm{BC}$ at $\zeta\left(z=l_{z}\right)$ was experimentally found to increase the kink instability threshold. In Fig. 3 for $\psi=22.5^{\circ}, 90^{\circ}$ we dis-

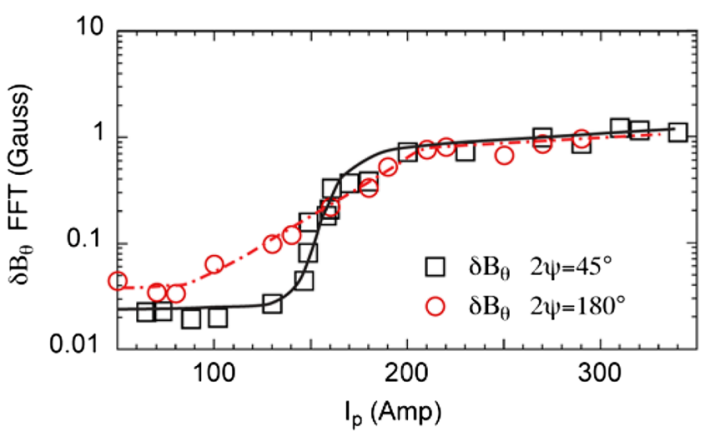

FIG. 3 (color online). Amplitude of the perturbed magnetic field $\delta B_{\theta}$ as function of plasma current $I_{p}$ with conical boundary $\psi=22.5^{\circ}$ and $90^{\circ}, B_{z}=100 \mathrm{G}$, showing thresholds, respectively, with abrupt and gradual transition to instability.

play plots of the magnetic fluctuations $\delta B_{\theta}$ measured outside the flux rope vs $I_{p}$. The changes in slope can be used to determine transitions between stable, growing, and saturation phases. For data near or below the $B_{\theta}$ instability threshold, for instance as in Fig. 3, the plasma motion can still be described by a linear dynamical model $[13,14]$. The kink threshold is $\sim 50 \%$ larger for $\psi=22.5^{\circ}$ than for $\psi=$ $90^{\circ}$. The growth in $\delta B_{\theta}$ with $I_{p}$ is more rapid for smaller cone angle, and saturates near $I_{p} \sim 200 \mathrm{~A}$ at $\delta B_{\theta} \sim$ 1-2 Gauss, or $1 \%-2 \%$ of the axial field strength $B_{z}$.

Figure 4 shows the kink instability threshold current $I_{p}$ for all six cones, where the KS threshold is indicated at $I_{p} \approx 155 \mathrm{~A}$ (line-tied) and the perfectly non-line-tied threshold is shown at $I_{p} \approx 78 \mathrm{~A}$. Threshold currents $I_{\text {th }}$ approach the KS limit with decreasing cone angle.

As suggested by Fig. 4 (and Fig. 5 later), the BC change at smaller cone angle stabilizes the kink instability and constrains the plasma motion on the boundary. It implies that the plasma flux rope becomes more line tied at smaller cone angle, which is consistent with a plasma resistive decay time in the sheath that becomes larger and comparable to the Alfven time. This situation would follow from

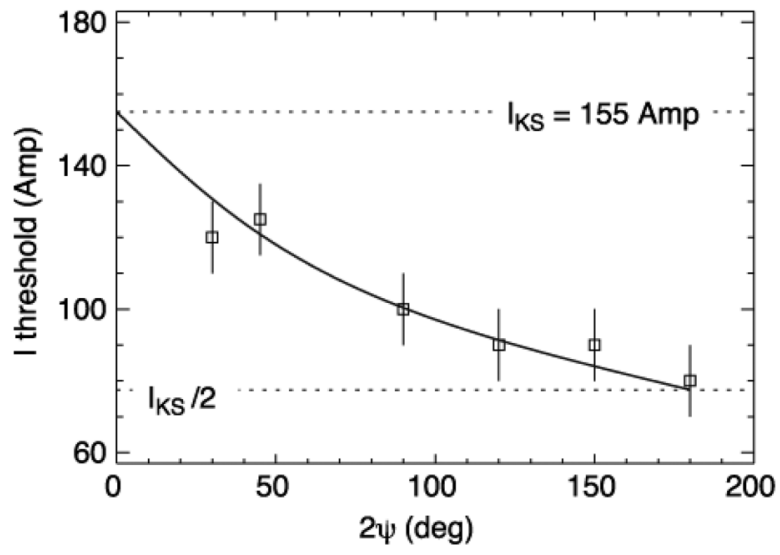

FIG. 4. Measured (squares) kink threshold flux rope current values of threshold current $I_{\text {th }}$ versus cone angles. Also indicated are threshold currents $I_{\mathrm{ks}}$ for line-tied and $I_{\mathrm{ks}} / 2$ for perfectlynon-line-tied conditions. 


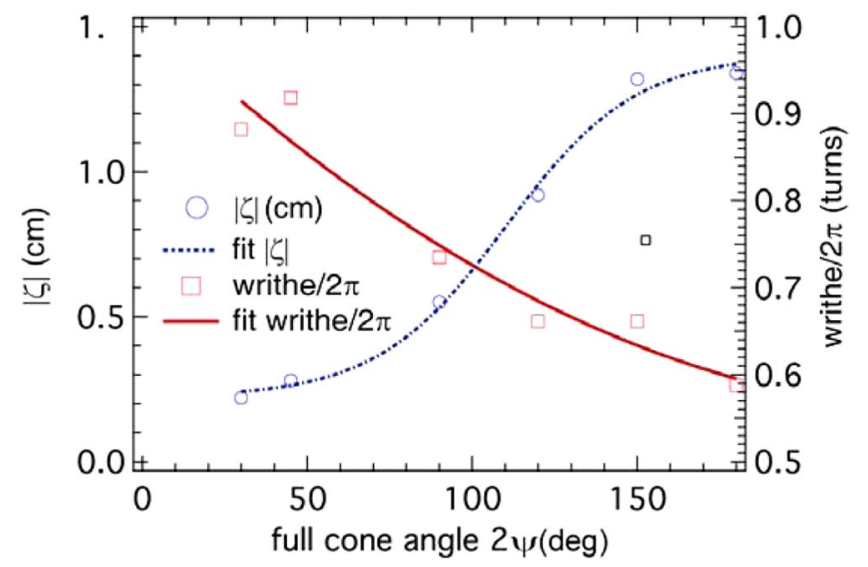

FIG. 5 (color online). Experimental displacements at the boundary $|\zeta|\left(z=l_{z}\right)$ indicated on the left hand axis, and corresponding turns $k_{0} l_{z} /(2 \pi)$ of writhe derived from the threshold current $I_{\text {th }}$, and flux rope radius $a=2.0 \mathrm{~cm}$, for a series of cone half angles $15^{\circ}<\psi<90^{\circ}$, varying from LT to NLT.

a reduced effective sheath resistance inversely proportional to $\sin (\psi)$, over the area of plasma that terminates on the endplate.

Independent optical images and more sensitive magnetic measurements provide further evidence that $\mathrm{BC}$ can be adjusted between PNLT and LT. As shown in the Fig. 1(a), a camera is used to image the plasma $H_{\alpha}$ emission from view 1 , and the displacement $\zeta(\psi)$ can be obtained directly from a series of shots and images such as Fig. 1(c). Experimentally measured magnetic fluctuations were shown to fit a theoretically predicted function $\delta B_{\theta}(\zeta)$ by Furno [17] for $\psi=90^{\circ}$, and we have also demonstrated this for other cone angles (data not shown here). We show plasma displacements $|\zeta|$ for a series of cone angles $(2 \psi)$ for $I_{p}=100 \mathrm{Amp}$ in Fig. 5 along with the writhe $w=k_{o} l_{z}$ number of turns, corresponding to $(r=a=2 \mathrm{~cm})$ at threshold current $I_{\text {th }}$ and $B_{z 0}$. A decrease in $\psi$ causes smaller plasma displacement (see Fig. 5) and enhances the line-tied property. Consistent with Fig. 4, the threshold $I_{\text {th }}$ thus approaches the KS limit.

In summary, the present experiments demonstrate for the first time that in linear geometry a shaped anode can be used to (1) change the kink properties without substantially changing most plasma parameters and (2) produce a continuous transition between line-tied and non-line-tied boundary conditions and the related MHD stability properties of the plasma. It has already been shown that a flat conducting boundary does not necessarily lead to a perfectly line-tied boundary condition [14]. The shaped anode acts like an adjustable constraint on plasma motion which can cause the line-tied condition, and the kink threshold current changes accordingly. This complements the usual cause and effect interpretation of line tying [8,9].

One might achieve a line-tied boundary by adding electron emission at the anode region to increase sheath conductivity. Aside from the kink threshold, other features of the Alfvén spectrum are also predicted to depend on the
BC $[1,23]$. From the perspective of mathematics and physics, this conclusion can be applied to many situations where the current-carrying plasma is nontoroidal, e.g., astrophysical jets, open flux embedded in the fast solar wind, and spheromak startup. Conversely, information about the BC could be inferred from other instability, threshold, or wave spectra measurements. This may be useful for observations of remote astrophysical systems, whose BC information is not directly accessible.

We gratefully acknowledge fruitful discussions and key ideas offered by D.D. Ryutov. Supported by U.S. Department of Energy through the LANL/LDRD Program.

[1] J.P. Goedbloed and G. Halberstadt, Astron. Astrophys. 286, 275 (1994).

[2] R. Tavakol, E. Covas, D. Moss, and A. Tworkowski, Astron. Astrophys. 387, 1100 (2002).

[3] C. R. Sovinec, J. M. Finn, and D. del-Castillo-Negrete, Phys. Plasmas 8, 475 (2001).

[4] J. H. Hammer and D. D. Ryutov, Phys. Plasmas 6, 3302 (1999).

[5] M. Zuin, R. Cavazzana, E. Martines, G. Serianni, V. Antoni, M. Bagatin, M. Andrenucci, F. Paganucci, and P. Rossetti, Phys. Rev. Lett. 92, 225003 (2004).

[6] T. Intrator, I. Furno, D. D. Ryutov, G. Lapenta, L. Dorf, and X. Sun, J. Geophys. Res. 112, A05S90 (2007).

[7] D. L. Meier, S. Koide, and Y. Uchida, Science 291, 84 (2001).

[8] M. A. Raadu, Sol. Phys. 22, 425 (1972).

[9] M. Gibons and D. S. Spicer, Sol. Phys. 69, 57 (1981).

[10] V. D. Shafranov, At. Energ. 5, 38 (1956).

[11] R. Kulsrud, in Plasma Astrophysics, edited by P. A. Sturrock (Academic, New York, 1967), p. 46.

[12] B. Lehnert, Phys. Fluids 9, 1367 (1966).

[13] D. D. Ryutov, I. Furno, T. P. Intrator, S. Abbate, and T. Madziwa-Nussinov, Phys. Plasmas 13, 032105 (2006).

[14] I. Furno, T. P. Intrator, D. D. Ryutov, S. Abbate, T. Madziwa-Nussinov, A. Light, L. Dorf, and G. Lapenta, Phys. Rev. Lett. 97, 015002 (2006).

[15] G. Lapenta, I. Furno, T. Intrator, and G. L. Delzanno, J. Geophys. Res. 111, A12S06 (2006).

[16] I. Furno, T. Intrator, E. Torbert, C. Carey, M. D. Cash, J. K. Campbell, W. J. Fienup, C. A. Werley, G. A. Wurden, and G. Fiksel, Rev. Sci. Instrum. 74, 2324 (2003).

[17] I. Furno, T. P. Intrator, G. Lapenta, L. Dorf, S. Abbate, and D. D. Ryutov, Phys. Plasmas 14, 022103 (2007).

[18] X. Sun, S. A. Cohen, E. E. Scime, and M. Miah, Phys. Plasmas 12, 103509 (2005).

[19] P. Stangeby, The Plasma Boundary of Magnetic Fusion Devices (IOP, Bristol, 2000), p. 81.

[20] R. H. Cohen and D.D. Ryutov, Phys. Plasmas 2, 2011 (1995).

[21] R. H. Cohen and D. D. Ryutov, Contrib. Plasma Phys. 44, 111 (2004).

[22] R. Chodura, Phys. Fluids 25, 1628 (1982).

[23] J.P. Goedbloed and S. Poedts, Principles of Magnetohydrodynamics (Cambridge University Press, Cambridge, England, 2004), p. 613. 